\title{
Revisión de los programas de sincronización ovárica basados en el uso de hormona liberadora de gonadotropinas y prostaglandina F2 $\alpha$ para novillas de leche y de carne
}

\author{
A. Sanz ${ }^{1,2}$, K. Macmillan ${ }^{1}$ y M.G. Colazo ${ }^{1, *}$ \\ 1 Livestock Systems Section, Alberta Agriculture and Forestry, Edmonton, Alberta, Canada \\ 2 Centro de Investigación y Tecnología Agroalimentaria de Aragón (CITA). Instituto Agroalimentario \\ de Aragón-IA2 (CITA-Universidad de Zaragoza), Zaragoza, España
}

\begin{abstract}
Resumen
La inseminación artificial lleva décadas implantada en las explotaciones de vacuno de leche, no tanto en el vacuno de carne. Los protocolos de sincronización ovárica que permiten realizar la inseminación artificial a tiempo fijo son muy interesantes, debido a que no dependen exclusivamente de la detección de celos, por lo que se han convertido en una herramienta muy útil para planificar y mejorar la eficiencia reproductiva de las explotaciones comerciales. El ganadero que quiera implementar estas técnicas reproductivas en su explotación deberá asesorarse por un especialista, que le recomiende los protocolos más adecuados para cada tipo de animal y para cada rebaño, teniendo en cuenta que los resultados obtenidos en novillas han sido más variables que en las vacas adultas. En este trabajo se revisan los protocolos de sincronización ovárica basados en el uso de hormona liberadora de gonadotropinas ( $\mathrm{GnRH}$ ) y prostaglandina F2 $\alpha$ (PGF) utilizados en novillas, así como los diversos ajustes que se han ido proponiendo, para tratar de unificar y mejorar las tasas de preñez.
\end{abstract}

Palabras clave: Recría, vacuno, reproducción, tratamientos, inseminación artificial a tiempo fijo.

A review of the ovarian synchronization programs based on the use of gonadotrophin releasing hormone and prostaglandin F2 $\alpha$ for dairy and beef heifers

\begin{abstract}
Artificial insemination has been utilized in dairy cattle for decades, but the adoption of this technique has been low in the beef industry. Ovarian synchronization protocols that allow for timed artificial insemination are attractive because estrus detection is not required, becoming a very useful tool to improve the overall reproductive efficiency of commercial herds. Producers should seek advice from a specialist, who can recommend the most appropriate protocols for each category of animal and for each herd, taking into account that the results obtained in heifers have been more variable than in adult cows. In this publication, we review the ovarian synchronization protocols based on gonadotrophin releasing hormone (GnRH) and prostaglandin F2 $\alpha$ (PGF) for dairy and beef heifers, as well as several modifications that have been proposed, to enhance pregnancy rates.
\end{abstract}

Keywords: Rearing, cattle, reproduction, timed artificial insemination.

* Autor para correspondencia: marcos.colazo@gov.ab.ca

Cita del artículo: Sanz A, Macmillan K, Colazo MG (2019). Revisión de los programas de sincronización ovárica basados en el uso de hormona liberadora de gonadotropinas y prostaglandina F2 $\alpha$ para novillas de leche y de carne. ITEA-Información Técnica Económica Agraria 115(4): 326-341. https://doi.org/10.12706/itea.2019.002 


\section{Introducción}

La eficiencia reproductiva de las novillas de recría es fundamental, puesto que su mantenimiento representa un elevado coste para la explotación antes de que paran un ternero y generen beneficios a la explotación. Las noviIlas inician la pubertad y comienzan a mostrar celos cuando su peso ronda el $45 \%$ (novillas de leche) o $55 \%$ de su peso adulto (novillas de carne), por lo que este factor no es un limitante a la hora de tener un primer parto a una edad temprana (dos años de edad).

La incorporación de la inseminación artificial (IA), ya sea para grupos selectos de animales o en el total del rebaño, mejora indudablemente las tasas de fertilidad de la explotación, puesto que, a las ventajas fisiológicas inherentes a los distintos tratamientos hormonales, hay que añadir que exige el cumplimiento de una serie de requisitos mínimos, como son la observación rutinaria de los animales y el registro continuado de sus rendimientos técnicos e incidencias sanitarias. El ganadero debe asesorarse por un especialista, que le recomiende los protocolos más adecuados para cada animal y cada rebaño. Otros requisitos imprescindibles a la hora de incorporar la IA en una explotación son: revisar los animales (ciclicidad y estado de carnes); la higiene en todos los procedimientos; la planificación del programa, productos, manga de manejo, etc.; el manejo de los animales sin estrés (evitar perros, gritos, vacunar o desparasitar durante el tratamiento o el mes siguiente, etc.); realizar diagnósticos de gestación para controlar el éxito de la IA, etc. Si se garantizan estos requisitos y la destreza del técnico o veterinario con el procedimiento de IA, el éxito está asegurado.

A pesar de su mayor coste económico, los protocolos para la inseminación artificial a tiempo fijo (IATF) se han extendido en gran medida en los últimos años, debido principalmente a que no es necesaria la detección de celos para llevar a cabo la IA. La detección de celos es el mayor factor limitante del éxito de los programas de IA, y en especial en las explotaciones extensivas. De ahí la idoneidad de los protocolos de IATF tanto para las novillas de leche como para las de carne. Además, se ha demostrado que los protocolos de IATF mejoran la eficiencia de la inseminación y reducen el intervalo entre el inicio de la época reproductiva y la primera IA, en comparación con la eficiencia obtenida al inseminar a las novillas que exhiben celo utilizando un protocolo de sincronización del estro (Silva et al., 2015).

\section{Tratamientos que sincronizan el crecimiento folicular y la ovulación}

Para poder realizar la IA de un grupo de animales a un tiempo determinado, y sin necesidad de detectar celo, es imprescindible sincronizar la función ovárica de las novillas, es decir, el crecimiento de los folículos presentes en los ovarios, la regresión del cuerpo lúteo $(\mathrm{CL})$ y la ovulación. En general, los tratamientos que se utilizan para sincronizar la función ovárica se basan en el uso de estradiol o de hormona liberadora de gonadotropinas $(\mathrm{GnRH})$, en ambos casos generalmente acompañado de un progestágeno. En la actualidad, el estradiol ya no está disponible para su uso en animales productores de alimentos en muchos países (Europa, USA, etc.), quedando restringida su aplicación a América del Sur y México (Canadá por ejemplo no tiene ninguna preparación de estradiol disponible en el mercado veterinario, obteniéndose únicamente con una prescripción médica). Por ello, en esta revisión nos centraremos en los tratamientos basados en el uso de la GnRH. Esta hormona induce la liberación de la hormona luteinizante (LH), la ovulación (en animales con un folículo dominante $\geq 10 \mathrm{~mm}$ de diámetro) y la emergencia de una nueva ola folicular dos días más 
tarde (Martínez et al., 1999). En 1995, Pursley et al. demostraron que el tratamiento con una prostaglandina F2 $\alpha$ (PGF) 6 o 7 días después de la primera GnRH y la aplicación de una $2^{\text {a }}$ dosis de GnRH entre las 36 y 48 horas después de la PGF permitía hacer una IATF entre 16 y 20 horas después de la administración de la $2^{\text {a }} \mathrm{GnRH}$. Este protocolo que combina el uso de GnRH y PGF para realizar una IATF se denomina Ovsynch. La mayoría de los protocolos de IATF que se utilizan hoy en día son variaciones del protocolo Ovsynch; por ejemplo, el protocolo Cosynch es una modificación del Ovsynch, utilizado más frecuentemente en vacuno de carne, en el que la $2^{a}$ dosis de GnRH se administra a la vez que se realiza la IATF, para reducir de 4 a 3 el número de manejos de los animales, y por tanto el coste del manejo reproductivo (Geary et al., 2001).

Tal y como recoge la bibliografía, las tasas de preñez obtenidas por IA en vacas lecheras tratadas con los protocolos Ovsynch de 7 días (7-d) han sido similares a las obtenidas en las vacas inseminadas a celo visto (38 vs. $39 \%$ ), no así en las novillas de leche (35 vs. 74\%, para Ovsynch e IA a celo visto; Pursley et al., 1997). Estos autores justificaron los peores resultados obtenidos en novillas debido al bajo porcentaje de ovulaciones detectadas después de la primera dosis de $\mathrm{GnRH}$, así como a la aparición posterior de un folículo dominante que no sería lo suficientemente maduro como para responder a la segunda inyección de GnRH (Pursley et al., 1997). Los resultados obtenidos por diversos autores con estos protocolos Ovsynch y Cosynch de 7 días, tanto en novillas de carne como de leche, han sido muy variables, e incluso contradictorios, por lo que se han ido estudiando diversos ajustes al protocolo clásico de 7 días, para tratar de unificar y mejorar los resultados de preñez en novillas. Se resumen a continuación las modificaciones propuestas más destacadas.
Incorporación de una progesterona a los protocolos Ovsynch/Cosynch de 7 días

Las primeras investigaciones realizadas con objeto de sincronizar los ciclos estrales en ganado vacuno ya proponían el uso de implantes subcutáneos del progestágeno norgestomet (Spitzer et al., 1976), para prevenir el estro y la ovulación. Posteriormente, numerosos estudios han confirmado que la colocación de un dispositivo intravaginal con progesterona (CIDR, Cue-Mate ${ }^{\circledR}$, DIB, PRID, etc.) ayuda a reducir la incidencia de celos prematuros. Martínez et al. (2002) observaron que la incorporación de un CIDR entre la primera GnRH y la administración de PGF del protocolo Cosynch 7-d aumentaba la tasa de preñez a la IATF (68 vs. 39\%) en novillas de carne. Estos resultados fueron confirmados posteriormente por Lamb et al. (2006), en un estudio que aglutinaba los resultados de 2.075 novillas de carne, procedentes de 12 explotaciones. Por su parte, DeJarnette et al. (2004) reportaron que la incorporación de un dispositivo intravaginal con progesterona en un protocolo tipo Ovsynch fue más efectivo en vacas de carne primíparas que en multíparas.

Richardson et al. (2002) realizaron un estudio para analizar las características de la expresión de celo previa a la IA, así como la tasa de preñez en novillas de leche y de carne sincronizadas usando una PGF precedida de una dosis de GnRH, con o sin CIDR. Los autores observaron que la adición del CIDR mejoró la proporción de animales que mostraban celo, tanto en las novillas de leche (79 vs. $74 \%$, con o sin CIDR, $\mathrm{P}<0,05$ ) como de carne (91 vs. $83 \%$, con o sin CIDR, $\mathrm{P}<0,05)$. Con respecto a la fertilidad, la inclusión del CIDR también aumentó la tasa de preñez en las novillas lecheras ( 46 vs. $35 \%$, con o sin CIDR, $\mathrm{P}<0,05$ ) y en las novillas de carne ( 53 vs. $49 \%$, con o sin (IDR), aunque esta última diferencia no 
fue estadísticamente significativa. Aunque puede encontrarse algún resultado no concluyente, en general, la inclusión de un dispositivo con progesterona en los protocolos de sincronización basados en el uso de GnRH y PGF en novillas se ha asociado a una reducción de las ovulaciones tempranas, y finalmente, a una mejora de la tasa de preñez.

\section{Duración del tratamiento con progesterona en los protocolos Ovsynch/Cosynch}

En 2008, Bridges et al. demostraron que reducir la duración del CIDR de 7 a 5 días, en un protocolo Cosynch + CIDR con IA a 60 o 72 horas, aumentaba la tasa de preñez en vacas de carne (60 vs. $70 \%$, respectivamente), debido probablemente a que el folículo dominante/ovulatorio se beneficiaría del soporte adicional de gonadotrofinas y de un tiempo extra para crecer y desarrollarse. Otros autores también han relacionado las ventajas del protocolo de 5 días (5-d) (menor incidencia de folículos persistentes, menor período de dominancia folicular y mayor duración del proestro) con un aumento de la tasa de preñez en vacas de carne (Whittier et al., 2013). Por su parte, Wilson et al. (2010), comparando un protocolo Cosynch 7-d + CIDR (IATF 66 horas después de la retirada del CIDR) frente a un Cosynch 5-d + CIDR (IATF 72 horas tras la retirada del CIDR), no observaron diferencias en la tasa de preñez en función del tratamiento, quedando finalmente preñadas el $67 \%$ de las vacas de carne. A raíz de estos resultados, los autores indicaron que la tasa de preñez quizá depende más de programar adecuadamente la IATF en relación con el proestro, que del momento de la retirada del CIDR o del desarrollo folicular. Es importante mencionar que el protocolo estándar de 5 días incluye una dosis extra de PGF que por lo general se administra entre 6 y 24 horas después de la primera dosis.
En cuanto a las novillas, hay pocos estudios publicados que comparen protocolos de sincronización basados en el uso de la GnRH y la PGF, combinados con una progesterona mantenida durante 7 o 5 días. Nuestro grupo realizó un estudio para comparar los protocolos Cosynch 7-d y 5-d en novillas lecheras (Colazo y Ambrose, 2011), usando una única dosis de PGF en ambos protocolos, en el que se obtuvo una tasa de preñez a la IATF similar en ambos protocolos (58 vs. 59\%, para 7 y 5 días, respectivamente), en línea con los resultados obtenidos más tarde por Lopes et al. (2013) (36 vs. $45 \%$, para 7 y 5 días). Estos últimos autores estimaron un coste económico $7 \$$ superior para cada novilla en el caso del protocolo 7-d, sobre todo debido a costes de semen, recría y remplazo. Posteriormente, Ahmadzadeh et al. (2015) también describieron una tendencia a mejorar la tasa de preñez en novillas de carne con el protocolo de 5-d + CIDR, frente al protocolo convencional de 7-d + CIDR (53 vs. $64 \%$, para 7 y 5 días, $P=0,07$ ).

Por tanto, la reducción de la duración del CIDR de 7 a 5 días en los protocolos de sincronización ha mejorado, o al menos no ha disminuido, la tasa de preñez en novillas de carne y de leche. Sin embargo, el coste de la terapia hormonal incluida en el protocolo estándar de 5-d es superior al de 7-d, de ahí que se hayan investigado otras modificaciones del protocolo de 5-d con el objetivo de disminuir el coste, manteniendo un alto porcentaje de preñez, para despertar el interés de los ganaderos de explotaciones comerciales.

\section{Eliminación de la segunda dosis de PGF de los protocolos Ovsynch/Cosynch de 5 días}

En los protocolos Ovsynch y Cosynch clásicos se administra una GnRH inicial para inducir la ovulación y el inicio de una nueva onda folicular. En el caso de los protocolos de 5 días, esta GnRH da lugar a un CL 2 días más joven 
al momento de administrar la PGF, comparado con los protocolos de 7 días. El nuevo $\mathrm{CL}$ que se forma tras la GnRH inicial puede no responder a un único tratamiento de PGF administrado 5 días después. De ahí que los autores que desarrollaron este protocolo se plantearan la necesidad de administrar una segunda dosis de PGF para garantizar la luteólisis (Bridges et al., 2008). Sin embargo, Rabaglino et al. (2010) examinaron el Cosynch 5-d + CIDR con 1 o 2 dosis de PGF en novillas lecheras, pero no encontraron diferencias significativas en la tasa de preñez a la IATF (53 vs. $59 \%$, para 1 o 2 dosis de PGF, respectivamente), concluyendo que un solo tratamiento con PGF sería suficiente para inducir la luteólisis en novillas sometidas a un Cosynch 5-d acompañado de un dispositivo de progesterona.

En el estudio realizado por nuestro equipo en el que se compararon los protocolos Cosynch de 7 y 5 días, se usó una única dosis de PGF en ambos protocolos (Colazo y Ambrose, 2011), alcanzando las novillas lecheras una tasa de preñez de $59 \%$ a la IATF, independientemente del protocolo aplicado. A pesar de administrarse una sola dosis de PGF en el protocolo de Cosynch 5-d, el $96 \%$ de las novillas presentaron regresión luteal. Estos resultados confirmaron que la segunda dosis de PGF no sería necesaria, por lo que podría eliminarse del protocolo, reduciendo así el número de manejos necesarios y el coste del protocolo de sincronización. Un estudio posterior que comparaba el uso de 1 o 2 dosis de PGF en novillas lecheras en un protocolo Cosynch 5-d tampoco detectó diferencias en la tasa de preñez a la IATF (Kasimanickam et al., 2014), apoyando la teoría de que la segunda PGF no es necesaria en novillas lecheras.

Otros estudios, sin embargo, han reportado una tendencia a mejorar la tasa de preñez en el lote de novillas de carne que había recibido dos dosis de PGF 6 horas después de la retirada del dispositivo de progesterona (Peterson et al., 2011). En la misma línea, Lima et al. (2013) observaron que la luteólisis de los $\mathrm{CL}$ recién formados aumentaba con una segunda PGF, aunque no tenía efecto sobre los $\mathrm{CL}$ más antiguos, concluyendo que el protocolo Ovsynch/Cosynch 5-d en novillas lecheras requeriría 2 dosis de PGF (Lima et al., 2013). En todo caso, estos autores describieron que solo el $34 \%$ de las novillas ovularon con la primera dosis de $\mathrm{GnRH}$, dando lugar a un nuevo $\mathrm{CL}$ en el momento de retirar el CIDR, lo que indicaría que la $2^{\text {a }}$ PGF no sería necesaria en el $66 \%$ de los animales.

\section{Eliminación de la primera dosis de GnRH de los protocolos Ovsynch/Cosynch}

Como hemos señalado anteriormente, la GnRH inicial incluida en los protocolos Ovsynch/ Cosynch solo es realmente efectiva para inducir la ovulación cuando se administra en presencia de un folículo dominante $\geq 10 \mathrm{~mm}$ de diámetro. En 2006, Lamb et al. (2006) describieron que la respuesta a la GnRH administrada al colocar el CIDR en un protocolo Cosynch 7-d era bastante limitada en el caso de las novillas, mejorando únicamente un 3$4 \%$ la tasa de preñez frente a los lotes que no recibieron la GnRH inicial. Por lo tanto, nuestro grupo se planteó la hipótesis de que la GnRH inicial no sería necesaria en novillas, lo que reduciría el coste del protocolo, en particular el de 5 días, ya que sin el uso de la GnRH inicial no sería tampoco necesario administrar una segunda PGF. Para comprobar dicha hipótesis, se realizaron varios estudios en novillas, aplicando un protocolo Cosynch 5 -d con (+) o sin (-) la GnRH inicial y con una sola dosis de PGF (Tabla 1). En el primer ensayo, realizado en novillas de leche (Colazo y Ambrose, 2011), únicamente un $25 \%$ de las novillas ovularon tras la GnRH inicial, obteniéndose finalmente una mayor tasa de preñez en las novillas que no ovularon a la primera GnRH (65 vs. 45\%, para novillas sin o 
Tabla 1. Programa diario de tratamientos para los protocolos Cosynch 7-d, Cosynch 5-d estándar (+GnRH y 2 PGF) y Cosynch $5-d$ modificado (-GnRH y 1 PGF).

Table 1. Daily injection schedule of the 7-d Cosynch, standard 5-d Cosynch (+GnRH \& 2 PGF) and modified 5-d Cosynch (-GnRH \& 1 PGF) protocols.

\begin{tabular}{|c|c|c|c|c|c|c|c|c|c|c|}
\hline Protocolo & Mar & Mie & Jue & Vie & Sab & Dom & Lun & Mar & Mie & Jue \\
\hline Cosynch 7-d & $\begin{array}{l}\text { (AM) } \\
\text { GnRH } \\
+ \text { DIP4 }\end{array}$ & & & & & & & $\begin{array}{l}\text { (AM) } \\
\text { PGF } \\
\text {-DIP4 }\end{array}$ & & $\begin{array}{l}\text { (PM) } \\
\text { GnRH } \\
\text { IATF }\end{array}$ \\
\hline $\begin{array}{l}\text { Cosynch 5-d } \\
+ \text { GnRH } \\
2 \text { PGF }\end{array}$ & & $\begin{array}{l}\text { (AM) } \\
\text { GnRH } \\
+ \text { DIP4 }\end{array}$ & & & & & $\begin{array}{l}\text { (AM) } \\
\text { PGF } \\
\text {-DIP4 }\end{array}$ & $\begin{array}{l}(\mathrm{AM}) \\
\mathrm{PGF}\end{array}$ & & $\begin{array}{l}\text { (AM) } \\
\text { GnRH } \\
\text { IATF }\end{array}$ \\
\hline $\begin{array}{l}\text { Cosynch 5-d } \\
\text { modificado } \\
\text {-GnRH } \\
1 \text { PGF }\end{array}$ & & $\begin{array}{c}(\mathrm{AM}) \\
+ \text { +DIP4 }\end{array}$ & & & & & $\begin{array}{c}\text { (AM) } \\
\text { PGF } \\
- \text { DIP4 }\end{array}$ & & & $\begin{array}{l}\text { (AM) } \\
\text { GnRH } \\
\text { IATF }\end{array}$ \\
\hline
\end{tabular}

+DIP4: inserción del dispositivo intravaginal que contiene progesterona.

-DIP4: retirada del dispositivo intravaginal que contiene progesterona.

GnRH: hormona liberadora de gonadotropina.

PGF: prostaglandina F2 $\alpha$.

IATF: inseminación artificial a tiempo fijo.

con ovulación tras la GnRH inicial, respectivamente). Además, no se observaron diferencias significativas debidas a la GnRH inicial sobre la tasa de preñez (68 vs. 71\%, para los lotes $+\mathrm{GnRH}$ y $-\mathrm{GnRH}$, respectivamente). Estos resultados se han confirmado en estudios posteriores, aplicando el mismo protocolo en novillas de leche y de carne (Macmillan et al., 2017; Colazo et al., 2018a; Tabla 2). En otro estudio reciente, realizado en novillas de carne (López-Helguera et al., 2018), tampoco encontramos diferencias significativas en la tasa de preñez a la IATF debidas al uso de la dosis inicial de $\mathrm{GnRH}$ (56 vs. 55\%, para los lotes $+\mathrm{GnRH}$ y $-\mathrm{GnRH}$, respectivamente), en línea con las tasas de preñez obtenidas por otros equipos en novillas de carne, al estudiar la inclusión o no de la GnRH al inicio de un protocolo de sincronización (51 vs. 55\%) (Cruppe et al., 2014).

Lima et al. (2013), por su parte, observaron una mayor tasa de preñez en novillas leche- ras tratadas con un protocolo con GnRH y dos dosis de PGF, frente al protocolo sin GnRH y con una única dosis de PGF (62 vs. 53\%, respectivamente). Sin embargo, Kasimanickam et al. (2014) no encontraron ningún efecto de la GnRH inicial sobre la fertilidad de novillas lecheras (51 vs. $54 \%$ ), pero sí en novillas de carne (60 vs. $50 \%$ ), utilizando un protocolo con 1 PGF. En este estudio, se observó que el uso de la GnRH inicial aumentaba la tasa de preñez cuando el folículo era $\geq 8 \mathrm{~mm}$, en comparación con el protocolo sin $\mathrm{GnRH}$ (70 vs. $47 \%$ ), lo que sugiere que un folículo dominante no eliminado en el momento de la inserción de CIDR dará lugar a un oocito envejecido y reducirá la fertilidad en novillas de carne. Estos resultados podrían explicar, al menos en parte, los resultados contradictorios que recoge la bibliografía en los estudios realizados con novillas.

Los estudios anteriores demuestran que las tasas de preñez obtenidas aplicando un pro- 
Tabla 2. Comparación de la tasa de preñez (\%) entre las diferentes modificaciones de los protocolos Cosynch 5-d en novillas de carne y de leche.

Table 2. Comparison of pregnancy per Al among different modifications of the 5-d Cosynch protocols in beef and dairy heifers.

\begin{tabular}{|c|c|c|c|c|c|}
\hline Estudios (tasas de preñez, \%) & $\mathrm{n}$ & $\begin{array}{c}+ \text { GnRH } \\
2 \text { PGF }\end{array}$ & $\begin{array}{c}+ \text { GnRH } \\
1 \text { PGF }\end{array}$ & $\begin{array}{c}-\mathrm{GnRH} \\
1 \mathrm{PGF}\end{array}$ & $P *$ \\
\hline Colazo y Ambrose, $2011^{1}$ & 56 & - & 68 & 71 & $>0,10$ \\
\hline Cruppe et al. $2014^{2}$ & 823 & - & 51 & 55 & $>0,10$ \\
\hline Lima et al. 2013, Exp. $1^{1}$ & 1106 & 59 & - & 54 & $<0,05$ \\
\hline Lima et al. 2013, Exp. $2^{1}$ & 2144 & 62 & - & 53 & $<0,05$ \\
\hline Kasimanickam et al. $2014^{1}$ & 1137 & 55 & 54 & 51 & $>0,10$ \\
\hline Kasimanickam et al. $2014^{2}$ & 1018 & 58 & 60 & 50 & $<0,05$ \\
\hline Macmillan et al. $2017^{1}$ a & 317 & - & 57 & 66 & $=0,08$ \\
\hline López-Helguera et al. 2018, General ${ }^{2}$ & 1068 & - & 56 & 55 & $>0,10$ \\
\hline López-Helguera et al. 2018, Cíclicas² & 852 & - & 57 & 62 & $>0,10$ \\
\hline López-Helguera et al. 2018, Acíclicas² & 216 & - & 48 & 35 & $<0,05$ \\
\hline Colazo et al. 2018a, Cíclicas² & 667 & - & - & 59 & - \\
\hline Colazo et al. 2018a, Cíclicas ${ }^{2}$ b & 664 & - & - & 48 & - \\
\hline Colazo et al. 2018a, Acíclicas² & 275 & - & 36 & - & - \\
\hline
\end{tabular}

GnRH: hormona liberadora de gonadotropina. PGF: prostaglandina F2 $\alpha$.

${ }^{1}$ Novillas lecheras, ${ }^{2}$ Novillas de carne. a $50 \%$ IA Semen sexado, b Semen sexado.

* Los valores $P$ indican una diferencia significativa $(<0,05)$, una tendencia a la diferencia $(0,05<P<0,10)$

o ninguna diferencia $(>0,10)$ entre los valores de tasa de preñez.

tocolo Cosynch 5-d sin GnRH inicial y con una sola dosis PGF han sido aceptables, tanto en novillas de carne como de leche, confirmando que la $\mathrm{GnRH}$ inicial es prescindible y podría evitarse este coste de los protocolos. Aunque los resultados de cada protocolo Cosynch 5-d sobre la fertilidad pueden ser variables, será importante que los veterinarios y los productores tengan en cuenta la mano de obra y el coste que conlleva cada tratamiento, a la hora de elegir el protocolo adecuado para cada explotación.

\section{Manejo de novillas acíclicas}

La falta de ciclicidad es un problema relativamente frecuente en los sistemas de pastoreo, tanto en novillas de carne como de leche. Es factible pensar que las novillas que no están cíclicas al comienzo de la época reproductiva tengan una fertilidad menor y más probabilidad de quedar vacías al final de la época reproductiva. Lamb et al. (2006) detectaron un $10 \%$ de novillas no cíclicas al inicio de un protocolo Cosynch 7-d, aunque estos autores no 
encontraron diferencias significativas en la tasa final de preñez en función de la ciclicidad inicial (57 vs. $59 \%$, para novillas púberes e impúberes, respectivamente), quizá debido a que el CIDR o la GnRH aplicados, o ambos, indujeron la ovulación y el reinicio de la ciclicidad.

En nuestro estudio de 2018 (López-Helguera et al.) se observó que un $21 \%$ de las novillas de carne no estaban cíclicas (226/1062), oscilando esta proporción de 8 a $27 \%$ entre los tres rebaños incluidos en el estudio. Las novillas que estaban acíclicas al inicio del tratamiento presentaron una menor probabilidad de quedar gestantes a la IA que las que estaban cíclicas (41 vs. 59\%). En este estudio, se compararon además los protocolos $+\mathrm{GnRH}$ y -GnRH con 1 PGF y, aunque no hubo ningún efecto sobre la fertilidad de las novillas cíclicas, el protocolo $+\mathrm{GnRH}$ sí que mejoró la tasa de preñez en las novillas acíclicas (48 vs. $35 \%$, para $+\mathrm{GnRH}$ y $-\mathrm{GnRH}$, respectivamente). También se observó que un tercio de las novillas acíclicas no recuperaron la ciclicidad después de la sincronización e IA, y aquéllas que se preñaron a la IATF tuvieron más probabilidades de perder la gestación (6 vs. $3 \%$ ). Al final de la época reproductiva (tras 2 rondas de IA y servicio natural durante 45 días más), la proporción de novillas vacías fue mayor dentro del grupo de las que no estaban ciclando al inicio del protocolo de sincronización (7 vs. $3 \%$ ). En novillas lecheras se han obtenido resultados similares, observando mayores tasas de preñez si las novillas estaban cíclicas al inicio de un protocolo Cosynch 5-d modificado (35 vs. 59\%, para acíclicas y cíclicas) (Colazo et al., 2018a). En la misma línea, los resultados de varios estudios realizados en Canadá, que aglutinan datos de más de 4000 IATF en novillas sometidas a un protocolo de sincronización de estradiol más progesterona de 7 días, mostraron que la fertilidad obtenida en la IA inicial fue un $10-15 \%$ inferior en las novillas acíclicas en comparación con las que estaban cíclicas al inicio de los tratamientos (Colazo, Resultados no publicados).

De todos estos resultados se desprenden dos posibles aplicaciones prácticas que los veterinarios y productores pueden utilizar para mejorar la fertilidad en novillas acíclicas. En primer lugar, los veterinarios podrían realizar ecografías ováricas antes de iniciar un protocolo IATF para identificar a las novillas acíclicas, y asignarles el protocolo $+\mathrm{GnRH}$. En segundo lugar, los productores podrían asignar todas las novillas al protocolo $+\mathrm{GnRH}$, manejo que favorecerá a las novillas acíclicas y no tendrá ningún efecto negativo sobre las cíclicas. La primera opción aumentaría los costes veterinarios, pero disminuirá la cantidad de GnRH utilizada, ayudando además a identificar novillas problemáticas, lo que mejorará aún más la fertilidad general del protocolo. Sin embargo, nuestros resultados ponen en tela de juicio si merece la pena usar protocolos de sincronización e IATF en novillas acíclicas, puesto que, aunque los protocolos que incluyen progestágenos favorecerán la ciclicidad en algunas novillas acíclicas y aumentarán sus posibilidades de quedar preñadas, la menor fertilidad y el mayor riesgo de perder la gestación afectarán negativamente a la rentabilidad de la implementación del programa de sincronización.

\section{Utilización de semen sexado}

El semen sexado puede ser muy útil para inseminar novillas, puesto que se reduce el riesgo de que las vacas primíparas sufran partos distócicos, y a su vez permite aumentar la tasa de mejora genética en el rebaño. Sin embargo, las tasas de preñez que se obtienen con semen sexado son inferiores a las del semen convencional, debido fundamentalmente a la menor concentración de espermatozoides, al daño que éstos sufren durante el proceso de selección y a la menor 
duración de la fertilidad óptima en el tracto reproductivo de la hembra (Seidel, 2014). Como la duración de la fertilidad óptima se reduce con el uso de semen sexado, es fundamental que la ovulación se produzca lo más cerca del momento de la IATF. Aunque en nuestro estudio de 2011 (Colazo y Ambrose) no se analizó el efecto del tipo de semen, se observó que el $75 \%$ de las novillas de leche que recibieron el protocolo de 5 días sin GnRH inicial ovularon en las 24 horas siguientes a la IATF, siendo esta tasa de ovulación mayor que la del protocolo con $\mathrm{GnRH}$ inicial, por lo que el protocolo de 5 días sin GnRH podría ser beneficioso en caso de usar semen sexado.

En este sentido, Macmillan et al. (2017) compararon los protocolos $+y-G n R H$ usando semen convencional o sexado en novillas de leche. Aunque la diferencia no fue estadísticamente significativa, se observó una mayor tasa de preñez en el caso de semen sexado combinado con el protocolo - GnRH en comparación con el protocolo $+\mathrm{GnRH}$ ( 60 vs. $50 \%$, respectivamente). Estos resultados sugieren que el protocolo Cosynch 5-d sin GnRH podría ser el método de elección para aplicar en novillas cuando se usa semen sexado, ya que el momento de la IA es más próximo a la ovulación cuando la IATF se realiza a las 72 horas de la retirada del dispositivo de progesterona. Otra opción para mejorar la tasa de preñez en novillas en el caso de utilizar semen sexado podría ser realizando la inseminación solamente a aquellos animales que a la IATF muestren estro después de una detección corta de celos.

\section{Inclusión de la detección de celo en los protocolos Ovsynch/Cosynch}

Aunque los protocolos de IATF no precisan la detección de celo, podría resultar interesante vigilar los celos de las novillas durante un periodo corto de tiempo tras la retirada del CIDR y antes de la IA, para tratar de mejorar la tasa de preñez. En el estudio de Colazo y Ambrose (2011), la tasa de preñez fue superior en las novillas lecheras que ovularon dentro de las 24 horas posteriores a la IATF, sugiriendo que dichos animales ovularon después de una liberación endógena de LH. Estudios posteriores han descrito mejoras en las tasas de preñez de los lotes de novillas que habían expresado celo, desde un $18 \%$ en novillas de carne (Ahmadzadeh et al., 2015) hasta un $27 \%$ en vacas de carne (Richardson et al., 2014), frente a los lotes de animales que no lo habían mostrado.

Colazo y Mapletoft (2017), trabajando en novillas de leche, incluyeron tres controles visuales de celos al día, durante 30 minutos cada uno. Los autores observaron un menor intervalo de tiempo entre la IA y la ovulación, y menos variable, en las novillas que fueron inseminadas a celo visto en comparación con las inseminadas a tiempo fijo ( $16 \pm 0,7$ vs. 21 $\pm 1,3$ horas), que estuvo asociado a una mayor tasa de preñez, especialmente en las novillas inseminadas con semen convencional (75 vs. $64 \%$, para novillas inseminadas a celo visto o a tiempo fijo). Los resultados de este ensayo mostraron que las novillas observadas en celo antes de la IATF con semen sexado tuvieron numéricamente el doble de probabilidades de quedarse gestantes frente a las que no expresaron celo, sin embargo, la diferencia entre los lotes no fue estadísticamente significativa.

Además, se ha observado que un $25-30 \%$ de las novillas que se sincronizan con un protocolo de 5 días sin GnRH inicial presentan celos entre las 36 y 48 horas después de la retirada del dispositivo de progesterona y un $80-90 \%$ de esas novillas ovulan antes de la IATF (Colazo y Ambrose, 2011; Colazo y Mapletoft, 2017). Por ello, nos hemos planteado la hipótesis de si el uso de una detección corta de celos antes de la IATF para la identifi- 
cación de animales idóneos para una IA temprana (56-60 horas después de la retirada del CIDR) aumentaría los porcentajes de preñez. En este sentido, Macmillan et al. (2017) realizaron una detección de celo a las 36 y 48 horas de la retirada del CIDR. Las novillas que mostraron celo se inseminaron a las 60 horas de la retirada del CIDR, frente a las que no expresaron celo, que se inseminaron a las 72 horas (IATF). La tasa de preñez de las novillas inseminadas a celo visto tendió a ser superior que la del lote IATF ( 68 vs. $58 \%, \mathrm{P}=$ $0,08)$. La tasa de celo, a su vez, fue mayor en las novillas sincronizadas con el protocolo $-\mathrm{GnRH}$ (22 vs. $14 \%$, $-\mathrm{GnRH}$ y $+\mathrm{GnRH}$, respectivamente). Esto podría explicar por qué la tasa de preñez global tendió a ser mayor en el protocolo - GnRH, en comparación con el protocolo $+\mathrm{GnRH}$ (66 vs. $57 \%$ ), y por qué la tasa de gestación fue numéricamente superior para el semen sexado en el protocolo-GnRH, como se ha mencionado en el apartado anterior.

En otro estudio, realizado en novillas de carne (Colazo et al., 2018a), se utilizaron parches Estrotect ${ }^{\mathrm{TM}}$ (colocados en la base de la cola, que indican que la novilla fue montada por otras novillas si la superficie plateada aparece raspada y pasa a tener un color visible) para detectar celo entre la retirada del CIDR y la IATF, aunque todas las novillas recibieron una IATF a las 72 horas de la retirada del CIDR. En función de la superficie de Estrotect ${ }^{\mathrm{TM}}$ frotada, se asignaba una puntuación a los parches de 0 (sin cambio de color), 1 ( $\leq 50 \%$ de cambio de color), 2 ( $>50 \%$ de cambio de color) y 3 (perdido). En general, la tasa de preñez fue superior en las novillas con una puntuación de 2 (61\%) y $3(59 \%)$, frente a las novillas con una puntuación de 0 $(32 \%)$ o $1(33 \%)$. Aunque la tasa de preñez tendió a ser mayor al usar semen convencional (52 vs. $48 \%, P=0,07)$, la mayor tasa de preñez que se obtuvo en aquéllas que mostraron un parche con una puntuación de 20
3 fue tanto con el semen convencional como con el sexado. Por lo tanto, la incorporación de parches para la detección de celos permitiría seleccionar a la IATF qué animales se inseminan con semen sexado y cuáles con semen convencional para así mantener una buena tasa de preñez global.

Por ello, recientemente se han realizado dos ensayos para analizar el posible beneficio de usar los parches Estrotect ${ }^{\mathrm{TM}}$ para identificar novillas idóneas para una IA temprana (56 horas tras la retirada del CIDR) con el objetivo de mejorar las tasas de preñez obtenidas con semen sexado (Colazo et al., 2018b). En el primer ensayo, aquellas novillas que no recibieron una IA temprana fueron inseminadas a las 72 horas con una administración de $\mathrm{GnRH}$ a las 56 horas o concurrente con la IATF. En el segundo ensayo, a las novillas que no recibieron una IA temprana se les aplicó una IATF a las 72 u 80 horas. En dichos estudios se ha observado que la identificación de animales idóneos para una IA temprana (56 horas) mejoró los porcentajes globales de preñez (63 vs. 55\%), y que la IATF en el resto de los animales (aquéllos no inseminados a las 56 horas) se podría realizar a las 72 u 80 horas de la retirada del dispositivo de progesterona. Aunque retrasar la IATF a las 80 horas aumentó un $6 \%$ la tasa de preñez, la diferencia no fue estadísticamente significativa. Sin embargo, la administración de GnRH a las 56 horas con IATF a las 72 horas no mejoró los porcentajes de preñez. En este ensayo, la detección de celo mediante el uso de parches ha sido muy útil para mejorar la tasa global de preñez con semen sexado, y a su vez podría disminuir el número de tratamientos con GnRH en los animales que exhiben celo previo a la IATF.

De los resultados anteriores se desprende la recomendación práctica de utilizar semen sexado únicamente en las novillas cuyos parches Estrotect ${ }^{\mathrm{TM}}$ muestren una puntuación de 203 , inseminando con semen convencional el 
resto de novillas, con el objetivo de aumentar la efectividad del semen sexado. Otra posible aplicación del uso de los parches sería para decidir el momento idóneo de realizar la IA. Retrasar el momento de la inseminación especialmente en las novillas que muestran parches con una puntuación de 0 o 1 a las 72 horas de la retirada del CIDR puede mejorar la tasa global de preñez en los rebaños. Además, la identificación de novillas en celo, ya sea antes o después de la IATF programada, permite eliminar los tratamientos con GnRH en el momento de la IA, y reducir por tanto el coste del protocolo de sincronización. A pesar del aumento de los costes de mano de obra y/o veterinarios, la incorporación de la detección de celo de las novillas en los protocolos de sincronización mejorará las tasas globales de preñez de los rebaños, especialmente en el caso de que se utilice semen sexado.

\section{Administración de eCG en los protocolos Ovsynch/Cosynch}

Aunque los programas de sincronización que combinan el uso de GnRH y PGF normalmente alcanzan resultados reproductivos óptimos, las tasas de preñez pueden verse alteradas en el caso de animales comprometidos metabólicamente (subnutrición, baja condición corporal, anestro posparto y vacas primíparas en crecimiento). En estas situaciones, el uso de la gonadotropina coriónica equina (eCG, originalmente denominada gonadotropina sérica de yegua gestante, PMSG) ha cobrado importancia, y especialmente en América del Sur. Esta glicoproteína presenta doble actividad tipo hormona foliculoestimulante (FSH) y $\mathrm{LH}$, por lo que administrada unas horas antes de la ovulación estimula el crecimiento folicular, aumenta el tamaño del folículo preovulatorio e incrementa las concentraciones plasmáticas de progesterona tras la ovulación, lo que explica que diversos trabajos ha- yan asociado la aplicación de 300 o 400 UI de eCG en el momento de retirar la progesterona en un protocolo de sincronización con mayores tasas de preñez en vacas tratadas durante el anestro posparto, criando a sus terneros (Baruselli et al., 2004; Bó et al., 2007).

Sin embargo, hay muy pocos estudios en Canadá y USA que se hayan centrado en el uso de la eCG en protocolos de sincronización en novillas, y los resultados existentes no son del todo concluyentes. En un estudio realizado con vacas y novillas de carne, en el que se aplicó un protocolo de 7 días que combinaba GnRH y PGF, la administración de eCG el día 7 tendió a mejorar la tasa de preñez, aunque únicamente en las vacas primíparas, animales con necesidades energéticas importantes (Small et al., 2009). En este trabajo, el uso de eCG no indujo un aumento del diámetro del folículo preovulatorio, como sí describió previamente Bó et al. (2007).

Por su parte, Menchaca et al. (2013), sincronizando animales de carne con un dispositivo intravaginal de progesterona acompañado de estradiol, obtuvieron una mejora en la tasa de preñez al usar eCG, siendo esta mejora estadísticamente significativa solo en las hembras que no presentaron un CL en el momento de la inserción de la progesterona (65 vs. $51 \%$, para vacas con o sin eCG; 44 vs. $32 \%$, para novillas con o sin eCG). Otros estudios no han podido mejorar las tasas de preñez mediante el uso de eCG en novillas de carne (Small et al., 2010), debido según los autores a que la mayoría de animales del ensayo presentaban folículos preovulatorios con un diámetro $\geq 10 \mathrm{~mm}$ en el momento de la IA, por lo que no tendrían comprometida la fertilidad.

Es importante señalar que algunos de los estudios que han observado mejores tasas de preñez al incorporar la eCG en los protocolos de sincronización de novillas incluían el uso de estradiol en dichos protocolos, y trabajaban además con animales con bajo estado 
corporal. En este sentido, nuestro grupo ha analizado el efecto de incorporar $300 \mathrm{UI}$ de eCG en el momento de retirar el CIDR en dos protocolos de sincronización corta (Colazo, Resultados no publicados), el Cosynch 5-d modificado (-GnRH inicial y 1 PGF) y el J-synch, un protocolo de 6 días que incluye benzoato de estradiol (Re et al., 2013). En este estudio, la administración de eCG ha aumentado la tasa global de estro en un $7 \%$ (68 vs. $75 \%$, sin o con eCG; $P<0,05$ ). Además, se ha detectado una mejora significativa de la tasa de preñez al incluir la eCG, aunque esta mejoría solo se ha observado en las explotaciones 2 y 3 del estudio
(Tabla 3), no en la explotación 1, resultado que podría deberse al hecho de que las novillas de esta última explotación no tuvieran comprometida su fertilidad al inicio del ensayo, como muestra su mayor tasa de preñez (59 vs. 48 vs. $43 \%$, para las explotaciones 1, 2 y 3 , respectivamente, $P<0,001$ ), en línea con la hipótesis de que la eCG sería útil únicamente en los animales que están comprometidos metabólicamente. El aumento detectado en la tasa de preñez al incorporar la eCG se ha observado además en los dos protocolos utilizados en este estudio (Cosynch modificado y Jsynch), como se muestra en la Tabla 3.

Tabla 3. Tasas de celo y de preñez en novillas de carne, en función del tipo de protocolo (Pr) (Cosynch 5-d modificado vs. J-synch), de la inclusión o no de eCG (eCG) y de la explotación (Ex).

Table 3. Estrus and pregnancy rate in beef heifers, according to the type of protocol (Pr) (modified 5$d$ Cosynch vs. J-synch), the inclusion or not of eCG (eCG) and the herd (Ex).

\begin{tabular}{|c|c|c|c|c|c|c|c|}
\hline & \multicolumn{2}{|c|}{ Cosynch 5-d } & \multicolumn{2}{|c|}{ J-synch } & \multicolumn{3}{|c|}{$P$ * } \\
\hline & $-e C G$ & $+e C G$ & -eCG & $+e C G$ & $\operatorname{Pr}$ & eCG & Ex \\
\hline \multicolumn{8}{|l|}{ Tasa de celo, \% } \\
\hline Explotación $1(n=400)$ & 61 & 73 & 67 & 67 & $>0,10$ & $<0,05$ & $<0,01$ \\
\hline Explotación $2(n=550)$ & 69 & 77 & 77 & 84 & & & \\
\hline Explotación $3(n=200)$ & 64 & 80 & 69 & 67 & & & \\
\hline Global $(n=1.150)$ & 65 & 77 & 69 & 73 & & & \\
\hline \multicolumn{8}{|l|}{ Tasa de preñez\#, \% } \\
\hline Explotación $1(\mathrm{n}=400)$ & 58 & 55 & 65 & 56 & $<0,05$ & $>0,10$ & $<0,001$ \\
\hline Explotación $2(n=550)$ & 38 & 50 & 43 & 57 & & & \\
\hline Explotación $3(n=200)$ & 32 & 45 & 49 & 51 & & & \\
\hline Global $(n=1.150)$ & 44 & 51 & 52 & 56 & & & \\
\hline
\end{tabular}

eCG: gonadotropina coriónica equina.

* Los valores $P$ indican una diferencia significativa $(<0,05)$, una tendencia a la diferencia $(0,05<P<0,10)$ o ninguna diferencia $(>0,10)$ entre los valores de tasa de preñez. " Interacción significativa eCG x Ex $(P<0,05)$. 


\section{Resincronización mediante el uso de los protocolos Ovsynch/Cosynch}

Es razonable pensar que si se consigue reducir el intervalo de tiempo entre la IA y la resincronización disminuirá el número de días vacíos de las novillas y aumentará por tanto la tasa general de preñez. En este sentido, Sinedino et al. (2014) analizaron los resultados obtenidos al realizar una resincronización temprana (iniciada 28 días tras la $1^{\mathrm{a}} \mathrm{IA}$, en vacas diagnosticadas no preñadas por sus niveles de PAG) o tardía (iniciada 46 días tras la $1^{\text {a }}$ IA, en vacas diagnosticadas no preñadas por palpación rectal). Para ello, después de realizar la $1^{\text {a }}$ IA (y asignar las vacas al azar al grupo temprano o tardío), las vacas que mostraban celo eran inseminadas ese mismo día. Estos autores inseminaron a celo visto un menor número de vacas vacías en el lote de resincronización temprana, antes del protocolo de resincronización asignado. Este lote de resincronización temprana presentó un menor intervalo entre inseminaciones, tanto en las realizadas a celo visto ( 22 vs. 28 días) como a tiempo fijo (35 vs. 55 días), pero la tasa final de preñez y el número de días abiertos fueron similares a los observados en el lote de resincronización tardía.

En nuestro estudio con novillas lecheras (Macmillan et al., 2017), se compararon 2 protocolos de resincronización con intervalos entre servicios de 35 o 42 días, analizando los protocolos con y sin GnRH en cada inseminación. En este estudio, se obtuvo una tasa de preñez acumulada del $81 \%$ después de 2 inseminaciones y del $97 \%$ después de 4 . Los grupos de resincronización de 35 y 42 días entre servicios presentaron una tasa de preñez similar (54 vs. 60\%), aunque se inseminaron más novillas a celo visto en el grupo de resincronización de 42 días que en el de 35 días ( 23 vs. $8 \%$, respectivamente; $P=0,05$ ), debido probablemente a que este protocolo finalizó cuando se esperaba que las novillas mostraran celo natural, 2 ciclos estrales después de la IA inicial.
En sistemas de producción de carne con una época de servicios corta, un intervalo entre servicios de hasta 35 días podría considerarse largo. Sin embargo, si la primera IA se realiza el primer día de la época reproductiva y 35 días después la segunda IA, es posible alcanzar tasas de preñez del $80 \%$ después de dos servicios en un período de 35 días. Además, si las novillas se mantienen con toros de repaso durante un ciclo estral extra, es factible conseguir una tasa de preñez acumulada del $90 \%$ al finalizar una época reproductiva de 63 días.

\section{Conclusiones}

De los estudios incluidos en esta revisión sobre los distintos protocolos de sincronización ovárica basados en el uso de GnRH y PGF utilizados en novillas de leche y de carne, se puede concluir que los protocolos Ovsynch y Cosynch de 7 días han mostrado resultados aceptables, aunque muy variables. Para tratar de unificar y mejorar las tasas de preñez, se han propuesto diversas modificaciones, como es la inclusión de un dispositivo intravaginal de progesterona, que se ha asociado a una reducción de las ovulaciones tempranas, y finalmente, a una mejora de la tasa de preñez. La reducción del protocolo Cosynch de 7 a 5 días, combinado con una progesterona, permite alcanzar tasas de preñez aceptables en novillas, e incluso mejorar las obtenidas con los protocolos clásicos de 7 días, aunque a un mayor coste de aplicación. Para reducir este coste, manteniendo un alto porcentaje de preñez, se han analizado otros ajustes, tratando de adaptar los protocolos a las necesidades de cada tipo de animal y de cada explotación. Se han observado mayores tasas de preñez en el protocolo Cosynch de 5 días con una GnRH inicial y dos dosis de PGF, especialmente en lotes con novillas cíclicas y acíclicas. Sin embargo, se han observado resultados similares en novillas cíclicas con el 
protocolo sin GnRH y con 1 sola PGF, lo que reduciría el trabajo y el coste asociados a su administración. También se han obtenido buenos resultados con el protocolo sin $\mathrm{GnRH}$ en el caso de utilizar semen sexado, especialmente si se realiza detección de celo en las novillas (mediante control visual o parches Estrotect ${ }^{\mathrm{TM}}$ ). Además, es posible aumentar el número de novillas inseminadas a celo visto si se respeta un intervalo de resincronización más largo, que finalice coincidiendo con el ciclo estral natural de las novillas. La detección de novillas con una puntuación de 0 en sus parches permitirá ajustar mejor el momento óptimo de IA, siendo recomendable en estos casos retrasarla para que tengan tiempo suficiente para entrar en celo y ovular lo más cerca de la inseminación. Finalmente, será imprescindible tener en cuenta los objetivos productivos del ganadero, la mano de obra disponible y los costes de los servicios veterinarios y de los tratamientos, antes de elegir el protocolo de sincronización más adecuado para cada explotación y cada tipo de animal.

\section{Agradecimientos}

Los estudios de los autores incluidos en este artículo se han financiado con el apoyo de Alberta Agriculture and Forestry, Vetoquinol NA Inc., Estrotect Inc., Intervet Canada Corp., Alta Genetics, ganaderos colaboradores de leche y carne de Alberta (Canadá), y proyectos INIA RTA2013059, INIA RZP2017-001 (España). Albina Sanz ha recibido una Subvención para Estancias de movilidad del Ministerio español de Ciencia, Innovación y Universidades (PRX18/00097).

\section{Referencias bibliográficas}

Ahmadzadeh A, Gunn D, Hall JB, Glaze JB Jr (2015). Evaluation of treatment with a 5-day versus 7day controlled internal drug-release insert on reproductive outcomes of beef heifers using a modified timed-artificial insemination protocol. The Professional Animal Scientist 31: 270-277.

Baruselli PS, Reis EL, Marques MO, Nasser LF, Bó GA (2004). The use of hormonal treatments to improve reproductive performance of anestrus beef cattle in tropical climates. Animal Reproduction Science 82-83: 479-486.

Bó GA, Cutaia L, Peres LC, Pincinato D, Maraña D, Baruselli PS (2007). Technologies for fixed-time artificial insemination and their influence on reproductive performance of Bos indicus cattle. En: Reproduction in Domestic Ruminants VI (Eds. Juengel JL, Murray JF and Smith MF), pp. 223-236. Nottingham University Press.

Bridges GA, Helser LA, Grum DE, Mussard ML, Gasser CL, Day ML (2008). Decreasing the interval between GnRH and PGF2 $\alpha$ from 7 to 5 days and lengthening proestrus increases timed-Al pregnancy rates beef cows. Theriogenology 69: 843-851.

Colazo MG y Ambrose DJ (2011). Neither duration of progesterone insert or initial GnRH treatment affected pregnancy per timed-insemination in dairy heifers subjected to a Co-Sync protocol. Theriogenology 76: 578-588.

Colazo MG y Mapletoft RJ (2017). Pregnancy per $\mathrm{Al}$ in Holstein heifers inseminated with sex-selected or conventional semen after estrus detection or timed-Al. Canadian Veterinary Journal 58: 365-370.

Colazo MG, Whittaker P, Macmillan K, Bignell D, Boender G, de Carvalho Guimaraes R, Mapletoft RJ (2018a). Evaluation of a modified GnRHbased timed-Al protocol associated with estrus detection in beef heifers inseminated with sexselected or conventional semen. Theriogenology 118: 90-95.

Colazo MG, Macmillan K, Mapletoft RJ (2018b). Modifications of a 5-d GnRH-based timed-Al protocol to optimize fertility in Holstein heifers inseminated with sex-selected semen. 10th International Ruminant Reproduction Symposium (IRRS), 16-20 Septiembre 2018, Foz do Iguazu, Brazil. Abstract 97. 
Cruppe LH, Day ML, Abreu FM, Kruse S, Lake SL, Biehl MV, Cipriano RS, Mussard ML, Bridges GA (2014). The requirement of $\mathrm{GnRH}$ at the beginning of the five-day Co-synch + controlled internal drug release protocol in beef heifers. Journal of Animal Science 92: 4198-4203.

DeJarnette JM, House RB, Ayars WH, Wallace RA, Marshall CE (2004). Synchronization of estrus in postpartum beef cows and virgin heifers using combinations of melengestrol acetate, $\mathrm{GnRH}$, and PGF2 alpha. Journal of Animal Science 82: 867-877.

Geary TW, Whittier JC, Hallford DM, MacNeil MD (2001). Calf removal improves conception rates to the Ovsynch and Co-synch protocols. Journal of Animal Science 79: 1-4.

Kasimanickam RK, Firth P, Schuenemann GM, Whitlock BK, Gay JM, Moore DA, Hall JB, Whittier WD (2014). Effect of the first GnRH and two doses of PGF2 $\alpha$ in a 5-day progesterone-based Co-Synch protocol on heifer pregnancy. Theriogenology 81: 797-804.

Lamb GC, Larson JE, Geary TW, Stevenson JS, Johnson SK, Day ML, Ansotegui RP, Kesler DJ., DeJarnette JM, Landblom DG (2006). Synchronization of estrus and artificial insemination in replacement beef heifers using gonadotropin-releasing hormone, prostaglandin $\mathrm{F} 2 \alpha$, and progesterone. Journal of Animal Science 84: 3000-3009.

Lima FS, Ribeiro ES, Bisinotto RS, Greco LF, Martinez $\mathrm{N}$, Amstalden M, Thatcher WW, Santos JEP (2013). Hormonal manipulations in the 5-day timed artificial insemination protocol to optimize estrous cycle synchrony and fertility in dairy heifers. Journal of Dairy Science 96: 7054-7065.

Lopes G Jr, Johnson CR, Mendonça LG, Silva PR, Moraes JG, Ahmadzadeh A, Dalton JC, Chebel RC (2013). Evaluation of reproductive and economic outcomes of dairy heifers inseminated at induced estrus or at fixed time after a 5-day or 7-day progesterone insert-based ovulation synchronization protocol. Journal of Dairy Science 96: 1612-1622.

López-Helguera I, Whittaker P, Behrouzi A, Mapletoft RJ, Colazo MG (2018). Effect of initial $\mathrm{GnRH}$ and time of insemination on reproduc- tive performance in cyclic and acyclic beef heifers subjected to a 5-d Co-synch plus progesterone protocol. Theriogenology 106: 39-45.

Macmillan K, Loree K, Mapletoft RJ, Colazo MG (2017). Short communication: optimization of a timed artificial insemination program for reproductive management of heifers in Canadian dairy herds. Journal of Dairy Science 100: 4134-4138.

Martínez MF, Adams GP, Bergfelt D, Kastelic JP, Mapletoft RJ (1999). Effect of LH or GnRH on the dominant follicle of the first follicular wave in heifers. Animal Reproduction Science 57: 23-33.

Martínez MF, Kastelic JP, Adams GP, Cook RB, Olsen WO, Mapletoft RJ (2002). The use of progestins in regimens for fixed-time artificial insemination in beef cattle. Theriogenology 57 : 1049-1059.

Menchaca A, Núñez R, Wijma R, García Pintos C, Fabini F, de Castro T (2013). How fertility can be improved in fixed-time Al programs in beef cattle. In Proceedings X Symposium on Animal Reproduction (ed. GA Bó and M Caccia), Institute of Animal Reproduction Cordoba (IRAC), Córdoba, Argentina. pp. 103-134.

Peterson C, Alkar A, Smith S, Kerr S, Hall JB, Moore D, Kasimanickam R (2011). Effects of one versus two doses of prostaglandin F2 alpha on Al pregnancy rates in a 5-day progesterone-based, CO-Synch protocol in crossbred beef heifers. Theriogenology 75: 1536-1542.

Pursley JR, Mee MO, Wiltbank MC (1995). Synchronization of ovulation in dairy cows using PGF2alpha and GnRH. Theriogenology 44: 915-923.

Pursley JR, Wiltbank MC, Stevenson JS, Ottobre JS, Garverick HA, Anderson LL (1997). Pregnancy rates per artificial insemination for cows and heifers inseminated at a synchronized ovulation or synchronized estrus. Journal of Dairy Science 80: 295-300.

Rabaglino MB, Risco CA, Thatcher MJ, Lima F, Santos JEP, Thatcher WW (2010). Use of a five-day progesterone-based timed Al protocol to determine if flunixin meglumine improves pregnancy per timed Al in dairy heifers. Theriogenology 73: 1311-1318. 
Re M, de la Mata JJ, Bó GA (2013). Synchronization of ovulation in dairy heifers using a shortened estradiol-based protocol that provides for a lengthened proestrus. Reproduction, Fertility and Development 26: 118.

Richardson AM, Hensley BA, Marple TJ, Johnson SK, Stevenson JS (2002). Characteristics of estrus before and after first insemination and fertility of heifers after synchronized estrus using $\mathrm{GnRH}$, PGF2 $\alpha$, and progesterone. Journal of Animal Science 80: 2792-2800.

Richardson BN, Hill S, Stevenson JS, Djira GD, Perry GA (2014). Meta-analysis of the effect of estrus expression before fixed-time Al on conception rates in beef cattle. Journal of Animal Science 92 (Suppl. 2): 701.

Seidel GE Jr (2014). Update on sexed semen technology in cattle. Animal 8: 160-164.

Silva TV, Lima FS, Thatcher WW, Santos JEP (2015). Synchronized ovulation for first insemination improves reproductive performance and reduces cost per pregnancy in dairy heifers. Journal of Dairy Science 98: 7810-7822.

Sinedino LDP, Lima FS, Bisinotto RS, Cerri RLA, Santos JEP (2014). Effect of early or late resynchronization based on different methods of pregnancy diagnosis on reproductive performance of dairy cows. Journal of Dairy Science 97: 4932-4941.
Small JA, Colazo MG, Kastelic JP, Erickson NE, Mapletoft RJ (2010). Effects of presynchronization and eCG on pregnancy rates to $\mathrm{GnRH}$-based, fixed-time artificial insemination in beef heifers. Canadian Journal of Animal Science 90: 23-34.

Small JA, Colazo MG, Kastelic JP, Mapletoft RJ (2009). Effects of progesterone pre-synchronization and eCG on pregnancy rates to $\mathrm{GnRH}$ based, timed-Al in beef cattle. Theriogenology 71: 698-706.

Spitzer JC, Miksch ED, Wiltbank JN (1976). Synchronization following norgestomet and 5 or 6 mg EV. Journal of Animal Science 43: 305 (Abstr.).

Whittier WD, Currin JF, Schramm H, Holland S, Kasimanickam RK (2013). Fertility in Angus cross beef cows following 5-day CO-Synch + CIDR or 7-day CO-Synch + CIDR estrus synchronization and timed artificial insemination. Theriogenology 80: 963-969.

Wilson DJ, Mallory DA, Busch DC, Leitman NR, Haden JK, Schafer DJ, Ellersieck MR, Smith MF, Patterson DJ (2010). Comparison of short-term progestin based protocols to synchronize estrus and ovulation in postpartum beef cows. Journal of Animal Science 88: 2045-2054.

(Aceptado para publicación el 11 de enero de 2019) 